\section{Spinal Muscular Atrophy Type 1 With Exon 8 Deletion and Bilateral Optic Atrophy}

Spinal muscular atrophy (SMA) is an autosomal recessive neuromuscular disease with incidence of 1 in 5-10,000 live births and is caused by homozygous deletion of exons 7 and 8 in the SMN1 gene [1]. Isolated exon 8 deletion has been reported in only one case series [2]. It is characterized by symmetrical proximal weakness; although, extraocular muscles are typically spared, there are a few case reports of associated external ophthalmoplegia [3,4] and one case report with optic atrophy [5]. We report a boy with SMA type 1 with optic atrophy due to isolated deletion of exon 8 of the $S M N$ gene.

A preterm, 34 week, male baby, with birthweight of $1700 \mathrm{~g}$ was born to a 24 year-old para 2 live 1 mother, by third degree consanguineous marriage. Baby was delivered by vaginal route, with delayed cry at birth, was born limp and apneic requiring positive pressure ventilation and chest compression. Mother had history of polyhydramnios with amniotic fluid index (AFI) of $29 \mathrm{~cm}$ in second trimester with decreased fetal movements. There was a history of sibling death of a male baby at 30 weeks of gestation born one and half years back. On examination, baby was alert with spontaneous eye opening, had long facies with bilateral temporal hollowing; pupils were mid-dilated and non-reacting to light. Tone was decreased in all limbs, power was 2/5 around hip joint and knee joint and 3/ 5 in bilateral elbow joints; contractures were present at elbow, ankle and foot. Tongue fasciculations were present and bilateral deep tendon reflexes were absent. Baby had bulbar palsy with bilateral optic disk pallor.

Baby was continued on mechanical ventilation and IV fluids and later was started on tube feeds. Baby had recurrent episodes of gastroesophageal reflux (GER). Investigations revealed serum creatine phosphokinase (total) of $150 \mathrm{IU} / \mathrm{L}$, tandem mass pectrometry (TMS) and urine gas chromatography ass pectrometry (GCMS) were normal. Muscle biopsy showed maintained fascicular architecture with variation in fibre size with focal fat infiltration and occasional muscle fiber degeneration. No dystrophic, neurogenic, congenital myopathic, atrophic changes were seen. Gene analysis for $S M N 1$ gene revealed exon 8 deletion. Baby remained ventilated in hospital with multiple extubation failures, and died on day 57 of life.

In SMA, there is homozygous mutation or deletion of the survival motor neuron 1 (SMN1) gene, located in the telomeric region of chromosome 5q13. SMN2, a gene that is similar to SMN1 is located in the centromeric region, determines the severity of illness [6]. Genetic alteration to the $S M N 1$ gene is responsible for a reduction in survival motor neuron (SMN) protein. The SMN2 gene only produces $25 \%$ of SMN protein and so does not completely compensate for the absence of SMN1 [6]. The lack of the SMN protein causes degeneration of alpha motor neurons in the ventral horn of the spinal cord.

According to the age of onset and clinical severity SMA is divided into three subtypes (types 1-3). The hallmark of SMA type 1 is severe, progressive muscle weakness and hypotonia and present by 6 months of age, with $95 \%$ of patients having signs and symptoms by 3 months $[5,6]$. Optic atrophy is an unreported association of SMA, with the exception of one case report [5]. Present case had history of product of consanguineous marriage with previous sibling death with similar features, with antenatal ultrasound suggestive of polyhydramnios, with typical presentation of SMA and atypical presentation of extraocular muscle involvement with optic atrophy.

In conclusion, isolated exon 8 deletion of the $S M N$ gene is a rarely reported and unusual mutation of SMA and optic atrophy is an unreported association.

\section{BIJAYLAXMI BEHERA* AND AJAY KUMAR Department of Pediatrics, Maulana Azad Medical College, New Delhi, India. *jollybubu2008@gmail.com}

\section{REFERENCES}

1. Bogari NM, Bogari FR, Rayes HH, Alqassimi NM, Balto HM, Dannoun A, et al. Molecular Genetic Diagnosis for a family with type 1 spinal muscular atrophy (SMA) via analysis of the survival motor neuron (SMN) gene. J Rare Dis Diagn Ther. 2015;1:21.

2. Gambardella A, Mazzei R, Toscano A, Annesi G, Pasqua A, Annesi F, et al. Spinal muscular atrophy due to an isolated deletion of exon 8 of the telomeric survival motor neuron gene. Ann Neurol. 1998;5:836-9

3. Pachter BR, Pearson J, Davidowitz J, Reuben R, Boal D, Carr $\mathrm{R}$, et al. Congenital total external ophthalmoplegia associated with infantile spinal muscular atrophy. Fine structure of extraocular muscle. Invest Ophthalmol. 1976;15:320-4.

4. Dubrovsky A, Taratuto AL, Martino R. Distal spinal muscular atrophy and ophthalmoparesis: A case with selective type 2 fiber hypotrophy. Arch Neurol. 1981;38:594-6.

5. Maiti D, Bhattacharya M, Yadav S. Isolated exon 8 deletion in type 1 spinal muscular atrophy with bilateral optic atrophy: Unusual genetic mutation leading to unusual manifestation? J Postgraduate Med. 2012,58;4:294-5.

6. Baioni MTC, Ambiel CR. Spinal muscular atrophy: Diagnosis, treatment and future prospects. Jornal de Pediatria. 2010;86:261-9. 